\title{
An audit of the CaesAid vacuum-assisted delivery cup compared with forceps delivery of the fetal head in caesarean section
}

\author{
N N Stolwijk, ${ }^{1}$ BA (Med); P R de Jong, ${ }^{2}$ MMed, FRCOG (London), FCOG (SA) \\ ${ }^{1}$ Department of Obstetrics and Gynaecology, University of Amsterdam and AMC Hospital, Amsterdam, the Netherlands \\ ${ }^{2}$ Department of Obstetrics and Gynaecology, University of Cape Town and Netcare Christiaan Barnard Memorial Hospital, Cape Town, South Africa
}

Corresponding author: P R de Jong (pdejong@medi.co.za)

\begin{abstract}
Background. Vacuum-assisted caesarean delivery may result in a quicker delivery of the fetal head than the use of forceps, and improve maternal and fetal outcomes. The new CaesAid vacuum-assisted delivery (VAD) cup was designed specifically for this use.

Objectives. To assess whether the CaesAid VAD cup influences the duration of fetal head delivery at caesarean section when compared with forceps, and whether there are differences in perioperative complications in the mother and fetus.

Methods. We carried out a retrospective clinical audit of 132 patients who underwent caesarean delivery at the Netcare Christiaan Barnard Memorial Hospital in Cape Town, South Africa, from April to November 2017, aided by either CaesAid VAD cup (C group, $n=67$ ) or forceps (F group, $n=65$ ).

Results. The uterine incision-to-delivery interval was significantly shorter $(p=0.001)$ in the $\mathrm{C}$ group than the $\mathrm{F}$ group (median 38 (interquartile range (IQR) 20) v. 60 (IQR 50) seconds, respectively). The maternal blood loss was lower in the C group than the F group $(250 \mathrm{~mL}$ v. $288 \mathrm{~mL} ; p=0.025)$. There was no significant difference in Apgar scores or admissions to the neonatal intensive care unit. Neonatal skin injuries were less common in the C group (no cases v. 8 in the F group; $p=0.004$ ).

Conclusion. The results of this audit suggest that the CaesAid VAD cup is a safe and efficient alternative to forceps for aiding the delivery of the fetal head at caesarean section. However, the routine use of vacuum cups is debatable. Further research could provide more insight into this procedure as a part of obstetric practice.
\end{abstract}

S Afr J Obs Gynae 2019;25(1):20-24. DOI:10.7196/SAJOG.2019.v25i1.1377

Caesarean delivery rates worldwide have been increasing, and this is especially the case in the private sector in South Africa (SA). ${ }^{[1]}$ As a result, small differences in the efficiency of the procedure become important. ${ }^{[2]}$ It is common obstetric practice to deliver the fetal head in caesarean delivery by means of fundal pressure and manual extraction. If the head is deeply engaged in the maternal pelvis difficulty with the free-floating head is not encountered. If the head is not yet engaged, as may be the case in elective caesarean section and emergency procedures in early stages of labour, manual extraction is more difficult. Forceps may be employed in these cases, but their routine use in instrumental vaginal delivery in SA is declining, and physicians are no longer comfortable working with them. In 2010 2011, the assisted-delivery rate for vaginal births in SA was $0.52 \%$ for vacuum assistance, and $0.15 \%$ for forceps. ${ }^{[3]}$ Therefore the use of vacuum delivery at caesarean section might arise as a potentially beneficial option for delivering the fetal head, especially in cases of a high-floating fetal head. This audit compared the use of the forceps with the CaesAid vacuum-assisted delivery (VAD) cup, a silicone cup specifically designed for use at caesarean delivery.

The use of vacuum cups at caesarean section is not a new concept. Solomons ${ }^{[4]}$ and Arad et al.$^{[5]}$ reported vacuum extraction to be both effective and relatively safe, in 1962 and 1980, respectively. However, these early studies found that using the vacuum cup resulted in a longer time to delivery of the fetus than the use of forceps, which can be at least partly attributed to the fact that the Malmström cup they used had a narrow diameter and was made of metal, which made the application of the cup more time-consuming. Pelosi and
Apuzzio $^{[6]}$ were the first to describe the use of soft silicone vacuum cups at caesarean section, and concluded that the procedure was effective and not injurious to mother or baby. These results were corroborated in a study by Boehm ${ }^{[7]}$ that included 44 cases, and additionally in studies by Bofill et al. ${ }^{[8]}$ Dimitrov et al. ${ }^{[9]}$ and Sritippayawan and Chantrapitak. ${ }^{[10]}$ Bofill et al.$^{\left[{ }^{[8]}\right.}$ found that delivery times were longer for forceps-assisted caesarean delivery than for vacuum extraction and manual delivery, in a randomised pilot study with 44 participants. Sritippayawan and Chantrapitak ${ }^{[10]}$ also found that the duration of delivery was significantly shorter in vacuumassisted deliveries of the fetal head, in a study that included 90 cases of caesarean sections.

Additionally, Bofill et al., Dimitrov et al. and Sritippayawan and Chantrapitak found no significant difference in Apgar scores when comparing vacuum, forceps and/or manual extraction of the fetal head, and no adverse neonatal events were reported. ${ }^{[8-10]}$ There is insufficient literature on instrumentally assisted caesarean section to provide certainty on the incidence of possible complications. Current guidelines of the National Institute for Health and Care Excellence (NICE), RCOG and ACOG do not offer a clear position on the use of a vacuum cup at caesarean section. Forceps are mentioned in the NICE guideline, which states that they should only be used in cases of difficulty delivering the fetus. ${ }^{[11]}$

We have designed a new vacuum device (the CaesAid VAD cup; Fig. 1) specifically for use at caesarean section. The cup is manufactured by Medela in Switzerland. Its key feature is its soft, translucent silicone elastomer with a $60 \mathrm{~mm}$ diameter cup; existing 
vacuum cups are larger, and therefore harder to manoeuver in the pelvis at caesarean delivery. A silicone hose attaches to an electric vacuum device (Fig. 2), and the cup is reusable. Initial clinical experience with the device was encouraging, and so we carried out a retrospective clinical audit to evaluate the efficiency and safety of the CaesAid VAD cup in comparison with the use of forceps for the delivery of the fetal head at caesarean section.

Our primary objective was to assess if the use of the CaesAid VAD cup shortens delivery time of the fetal head when compared with forceps-aided delivery at caesarean section, as the literature

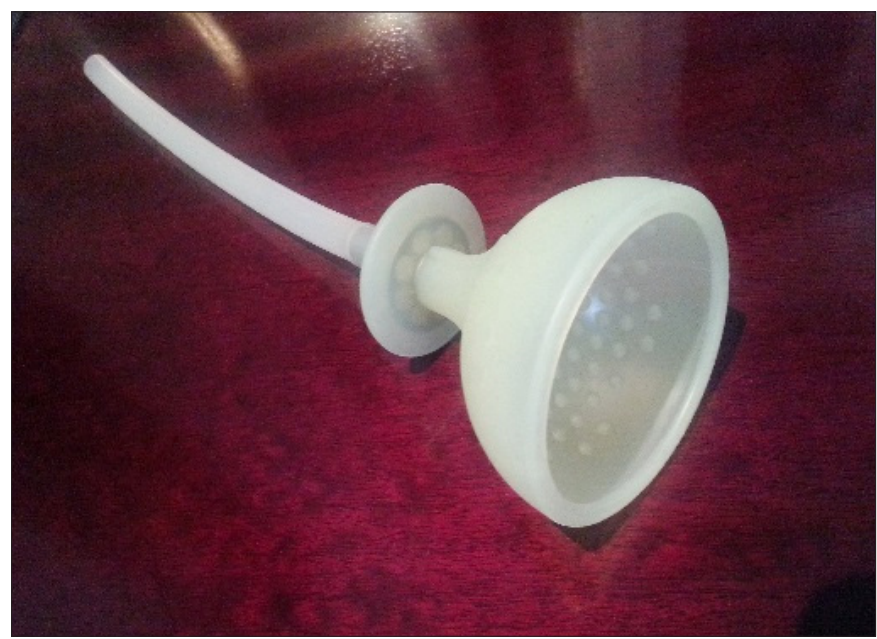

Fig. 1. CaesAid VAD cup.

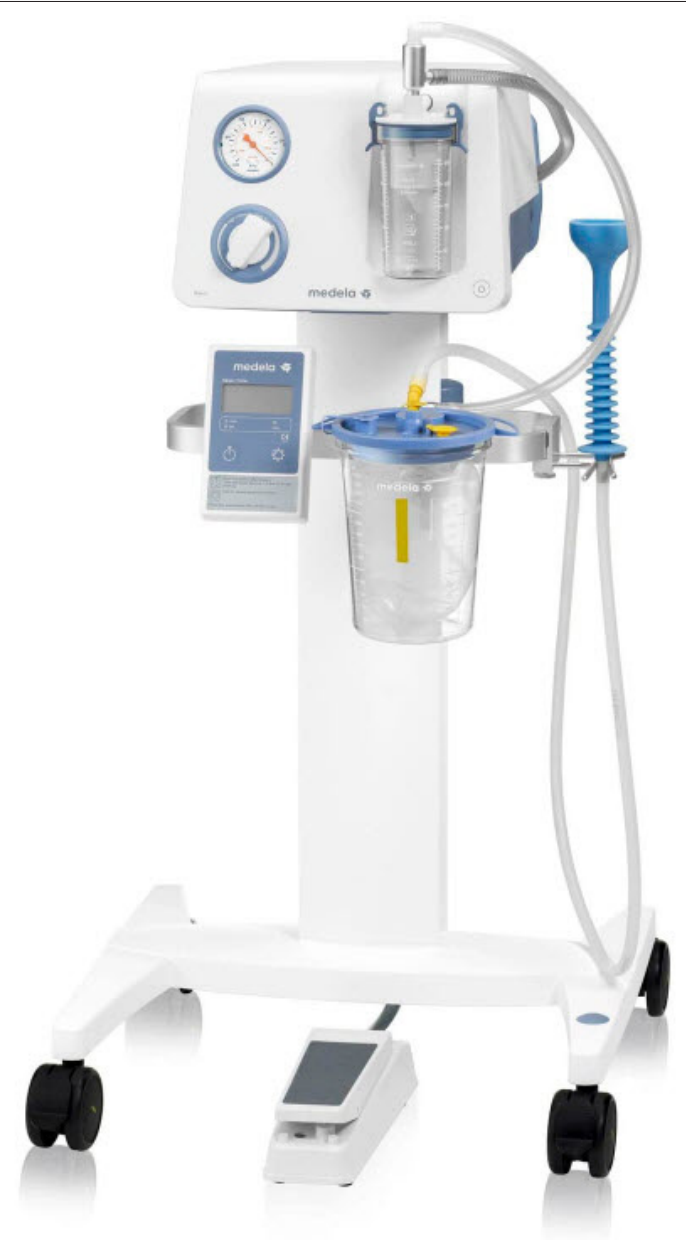

Fig. 2. Electric vacuum pump. on this topic is sparse, and the reported outcomes differ. Increased time to delivery of the fetal head is associated with compromised maternal and fetal outcomes and more complications. ${ }^{[12,13]}$ The secondary objective was to assess whether there was a difference in perioperative complications in the mother and fetus between the two methods of delivery.

\section{Methods}

A total of 132 patients underwent caesarean section aided by either the CaesAid VAD cup or forceps at the Netcare Christiaan Barnard Memorial Hospital, a private hospital in Cape Town, SA, between April and November 2017. Using the primary outcome measure of uterine incision to time to delivery of the fetal head (uterine incision-to-delivery interval; U-D interval), we anticipated a $20 \%$ difference in the time to delivery using the CaesAid VAD cup compared with forceps delivery: forceps delivery takes approximately 40 seconds, and we anticipated a $20 \%$ reduction in delivery time to 32 seconds using the CaesAid VAD cup. A power calculation to detect differences using a one-sided independent $t$-test with a $95 \%$ confidence interval, assuming that the actual distribution is normal, achieved $80 \%$ power for a population size of 120 participants. The study protocol was approved by the Pharma-Ethics Independent Research Ethics Committee (ref. no. 171119013).

All emergency and elective caesarean sections were carried out by two surgeons who had previously completed a training period of 10 caesarean sections using the CaesAid VAD device. While emergency v. elective caesarean section refers to the indication for the procedure as a whole, the elective use of the instruments refers only to choosing to use these as a first option to deliver the fetal head. All patients received spinal anaesthesia. Cases with a noncephalic presentation were excluded. Cases were selected on an approximately alternate case basis, without randomisation. Cases of prematurity were excluded.

At caesarean section, a lower uterine segment transverse incision was made, and the membranes were ruptured. The surgeon ascertained the fetal head position before applying the CaesAid VAD cup or forceps. The vacuum pressure used was $450 \mathrm{mmHg}$. If slight slippage occurred, the electric vacuum pump automatically restored the suction, diminishing the possibility of cup detachment (pop-offs). The forceps used were Wrigley's. If the CaesAid VAD cup or forceps delivery did not succeed after two attempts, the surgeon used an alternative device or manual extraction with fundal pressure. If the fetal head presented at the uterine incision, it was easily delivered by gentle fundal pressure and manual removal.

The U-D interval was timed by the anaesthetist with a stopwatch. Maternal blood loss was estimated by the anaesthetist based on the amount of blood collected by the intraoperative suction device and swab count. Ease of delivery was indicated on a 10-point scale by the surgeon ( 1 being the highest level of ease, and 10 the highest of difficulty). The skin incision was measured using a sterile ruler. The presence of any complications, and the need for a blood transfusion postoperatively were also noted. The paediatrician noted the Apgar scores and birth weight of the infant. Any evidence of neonatal trauma was documented. Additionally, the length of the infant's hospital stay, whether admission to the neonatal intensive care unit (NICU) was needed, and the length of the NICU stay, were recorded. The age, body mass index (BMI) and parity of the mother, 
the mode of previous delivery (if applicable) and the indication for caesarean section were gathered using the patient's hospital file.

The data were captured and analysed statistically using SPSS 25 (IBM Corp., USA). Data sets were compared using descriptive and comparative techniques. The normality of the data distribution was analysed by assessing the frequency distribution. For continuous variables, normally distributed data were reported using means (standard deviations (SD)), with differences examined using Student's independent $t$-test. Non-normally distributed data were reported as medians (interquartile ranges (IQR)), and differences examined using the Mann-Whitney $U$ test. Categorical data were reported as frequencies, and differences examined using the $\chi^{2}$ and Fisher's exact tests.

\section{Results}

The data of the 132 patients who underwent an elective or an emergency caesarean section in the period from April to November 2017 were analysed. The caesarean deliveries were aided by either the CaesAid VAD cup (C group, $n=67$ ) or forceps (F group, $n=65$ ). The maternal demographic factors in the two groups are shown in Table 1. Patients' age ranged from 20 to 43 years in the $\mathrm{C}$ group, with a mean (SD) of 32.5 (4.9), and from 23 to 42 in the F group, with a mean of 32.7 (4.5) in the F group. Patient BMI ranged from 19.6 to 48.2 in the C group, with a mean (SD) of 30.6 (8.4), and from 19.3 to 46.2 in the F group, with a mean (SD) of 31.9 (7.7). Table 1 also shows that maternal demographics were equivalent for both groups, except that fewer women in the F group had undergone a previous caesarean section.

Table 2 demonstrates that the U-D interval (the time of the incision into the uterus to the full delivery of the fetal head, in seconds) was significantly shorter in the C group $(p=0.001)$.
Additionally, a significant difference in the ease of delivery experienced by the primary surgeon was found, in that the F group showed greater variation in the ease of delivery scores $(p=0.001)$. The total operation time was also shorter in the $\mathrm{C}$ group $(p=0.045)$.

Slippage or folding of the vacuum cup occurred in 5 cases in total, with the cup folding in $3(4.5 \%)$ instances, and slipping once in 2 cases $(3.0 \%)$. In 25 cases the method of fetal head delivery differed from the intended intervention (see Table 2). In 8 of the 20 such cases in the F group, the method used to deliver the fetal head was altered owing to a spontaneous delivery of the head upon uterine incision. In the other 12 cases, the CaesAid VAD cup was employed after failure to deliver the fetal head with forceps. In the $\mathrm{C}$ group, there were 5 cases where a change in the delivery method of the fetal head occurred. Four of these were the result of spontaneous delivery of the fetal head upon uterine incision. In the remaining case, the vacuum cup became unsterile in theatre before use, and a forcepsaided fetal head delivery was performed.

As shown in Table 3, the estimated maternal blood loss was found to be significantly lower in the $\mathrm{C}$ group $(p=0.025)$, while the length of the skin incision did not differ significantly between the two groups. None of the mothers required a blood transfusion. No cases of deliberate or inadvertent extension of the uterine incision were reported. No significant difference in the length of hospital stay was found, with all mothers included in this trial being discharged within 3 days postoperatively.

The Apgar scores were equivalent in both groups (Table 4). There were no significant differences in the percentage of newborns that required specialised care in the NICU. Seven cases of NICU admission were reported in this audit. In most cases this was due to transient tachypnoea of the newborn, and in one case meconium

Table 1. Maternal demographic factors*

\begin{tabular}{|c|c|c|c|}
\hline Demographic factor & F group $(n=65)$ & C group $(n=67)$ & $p$-value \\
\hline Maternal age (years), mean (SD) & $32.7(4.5)$ & $32.5(4.9)$ & 0.308 \\
\hline BMI $\left(\mathrm{kg} / \mathrm{m}^{2}\right)$, median (IQR) & $30.6(10.9)$ & $28.1(13.4)$ & 0.391 \\
\hline Parity, median (IQR) & $1.0(1.0)$ & $1.0(1.0)$ & 0.084 \\
\hline Previous CS, $n(\%)$ & $23(34.3)$ & $33(50.8)$ & $0.008^{+}$ \\
\hline Emergency CS, $n(\%)$ & $25(37.3)$ & $16(24.6)$ & 0.115 \\
\hline
\end{tabular}

Table 2. Intraoperative outcomes ${ }^{*}$

\begin{tabular}{|c|c|c|c|}
\hline Outcome & F group $(n=65)$ & $C$ group $(n=67)$ & $p$-value \\
\hline U-D interval (s), median (IQR) & $60.0(50)$ & $38.0(20)$ & $0.001^{\dagger}$ \\
\hline Total operation time (min), median, IQR & $18(5)$ & $16.0(6)$ & $0.045^{\dagger}$ \\
\hline Ease of delivery, median (IQR) & $2(3)$ & $2(2)$ & $0.001^{\dagger}$ \\
\hline Change of method, $n(\%)$ & $20.0(30.8)$ & $5.0(7.5)$ & $0.001^{\dagger}$ \\
\hline
\end{tabular}

Table 3. Maternal outcomes ${ }^{\star}$

\begin{tabular}{lll}
\hline Outcome & F group $(\boldsymbol{n}=\mathbf{6 5})$ & C group $(\boldsymbol{n}=\mathbf{6 7})$ \\
\hline Maternal blood loss $(\mathrm{mL})$, median (IQR) & $288(100)$ & $250(110)$ \\
Skin incision length $(\mathrm{cm})$, median (IQR) & $13(2)$ & $13(2.5)$ \\
*Data analysed using Mann-Whitney U test. & & $0.025^{\dagger}$ \\
'Statistically significant. & & 0.441 \\
IQR = interquartile range. & &
\end{tabular}


RESEARCH

Table 4. Neonatal outcomes*

\begin{tabular}{|c|c|c|c|}
\hline Outcome & F group $(n=65)$ & C group $(n=67)$ & $p$-value \\
\hline Birth weight (g), median (IQR) & $3115(508)$ & $3340(645)$ & 0.092 \\
\hline 1-minute Apgar median (IQR) & $9.0(1.0)$ & $9.0(0)$ & 0.139 \\
\hline 5-minute Apgar median (IQR) & $10.0(1.0)$ & $10.0(0)$ & 0.421 \\
\hline NICU admission, $n(\%)$ & $5(7.5)$ & $2(3.1)$ & 0.238 \\
\hline Cephalhaematoma, $n(\%)$ & $1.0(1.6)$ & $5(8.2)$ & 0.207 \\
\hline Skin injury, $n(\%)$ & $8(13.3)$ & $0(0)$ & $0.004^{\dagger}$ \\
\hline
\end{tabular}

aspiration. While cephalhaematoma occurred more often in the C group, this difference was not found to be statistically significant ( $p=0.207)$. Skin injuries, consisting of skin lacerations, bruising and transient impressions, were found more frequently in the $\mathrm{F}$ group, and this difference was significant $(p=0.003)$. Of the eight cases where skin injuries occurred, three cases of skin laceration were reported. In three cases bruising occurred, and in the remaining two cases a transient impression on the skin was seen. No serious adverse neonatal events occurred during this audit.

\section{Discussion}

The primary aim of this retrospective clinical audit was to assess whether the CaesAid VAD cup significantly influences the time of fetal head delivery when compared with forceps-aided caesarean delivery. The results show that the U-D interval was significantly shorter for CaesAid VAD cup-assisted caesarean sections than for forceps-assisted deliveries. This is in line with the findings of previous small studies on this topic. ${ }^{[8-10]}$ A shorter U-D interval is desirable, since it is associated with improved maternal and fetal outcomes and fewer complications. ${ }^{[12,13]}$ The findings of this study refute an older study and one case report that suggested that vacuumassisted caesarean section results in longer times to delivery. ${ }^{[5,14]}$

The secondary aim was to examine whether there were differences in perioperative complications in the mother and fetus when comparing the CaesAid VAD cup with forceps. This audit found a decrease in maternal and neonatal complications for the vacuum cup in comparison with forceps-aided caesarean deliveries. Maternal blood loss was found to be lower when the vacuum cup was used. Previous research has found mixed results regarding maternal blood loss in vacuum-assisted caesarean sections, with results ranging from a non-significantly higher amount of maternal blood loss, to no difference or less blood loss. ${ }^{[4,5,8,10]}$

With regard to neonatal outcomes, the lack of difference in resulting Apgar score between the two instruments used is in line with the findings of the studies comparing vacuum-assisted caesarean section with either manual or forceps-aided delivery at caesarean section discussed previously. ${ }^{[8-10]}$ There is little research on the complications of the vacuum cup or forceps in caesarean section specifically, but some studies have looked at the use of these instruments in vaginal delivery. They show that cephalhaematoma occurs more often when the vacuum cup is used, and although the same was true in the present audit, this difference was not statistically significant. ${ }^{[15-17]}$ The studies also support the findings of this audit regarding the higher prevalence of skin injuries (lacerations and bruising) with the use of forceps.

There is controversy over the use of the vacuum cup in caesarean delivery. Vacuum-assisted caesarean delivery is routinely practised by a few private obstetricians in SA. One alarming case report from the USA argues that the routine use of vacuum cups for elective caesarean section is not justified. Clark et al. ${ }^{[14]}$ reported a case of subgaleal haemorrhage following elective vacuum-assisted caesarean section. It should be emphasised that in this case, there were two sudden disengagements of the vacuum cup (pop-offs), and the fetus was delivered on the third application of the vacuum cup. This practice is discouraged because of the risks illustrated in the case report. Existing literature states that the use of the vacuum cup should be discontinued after the occurrence of two pop-offs. ${ }^{[18]}$ The Clark report argued that use of the vacuum resulted in a prolonged time to delivery, but the findings of our audit and several other studies show the opposite. ${ }^{[5-7]}$ We found the use of the vacuum cup to be safe and efficient. In elective caesarean deliveries the vacuum cup might be especially useful for engaging a high-floating fetal head. Caution with the reapplication of vacuum cups should be urged when they are employed in a caesarean section, to prevent the occurrence of subgaleal haemorrhage. Moreover, the level of clinical experience needed for successful use of the vacuum cup should not be underestimated. ${ }^{[18,19]}$

Our audit has some limitations. As a retrospective study, the interventions were not randomised. Additionally, the ease of delivery was measured on a 10-point scale rather than the more commonly used Likert scale, with either 5 or 7 points. Finally, the maternal blood loss recorded was an estimate. An alternative means to determine blood loss during the procedure would be the weighing of swabs, drapes and other materials before and after the caesarean delivery, but this was not done in routine clinical practice. Measurement of the pre- and postoperative $\mathrm{Hb}$ to determine if there were differences in blood loss would be a useful addition in a prospective study.

To better our understanding of the use of soft vacuum cups such as the CaesAid VAD cup in caesarean delivery, further research is necessary. In 2018, a randomised trial was commenced at the Netcare Christiaan Barnard Memorial Hospital, comparing the CaesAid VAD cup with forceps-assisted delivery of the fetal head in caesarean section. Vacuum-assisted caesarean section seems most suitable for use in elective procedures where the fetal head is not yet deeply engaged in the pelvis and manual extraction might prove more difficult. If the potential benefits of vacuum extraction are substantiated by further research, there may be a place for the use of the CaesAid VAD cup in routine obstetric practice, especially in cases where the fetal head is inaccessible to conventional delivery.

Acknowledgements. The authors thank Prof. Philip Steer for his valuable comments, Roberta Abrahams for her secretarial help and Dr Erika Drewes for her support. 


\section{RESEARCH}

Author contributions. Equal contributions.

Funding. This study was funded by the Cape Town Pelvic Floor Foundation.

Conflicts of interest. PdeJ visited Medela's headquarters in Switzerland to discuss the design of the CaesAid VAD cup. This 2-day trip was funded by Medela. Other than this, he has not received any gain (monetary or otherwise) from his design. Additionally, he does not hold the patent for the design and declares no financial interest in Medela. NNS declares no conflicts of interest.

1. Naidoo R, Moodley J. Rising caesarean section rates: An audit of caesarean sections in a specialist private practice. S Afr Fam Pract 2009:51(3).254-258. https://doiorg/10.1080/20786204.2009.10 873857

2. The CORONIS Trial. International study of caesarean section surgical techniques: A randomised fractional, factorial trial. BMC Pregn Childbirth 2007;7(1):24. https://doi.org/10.1186/1471-2393-7-24 3. Pattinson RC, Vannevel V, Barnard D, et al. Failure to perform assisted deliveries is resulting in an increased neonatal and maternal morbidity and mortality: An expert opinion. S Afr Med 2018;108(2):75. https://doi.org/10.7196/samj.2017.v108i2.12786

4. Solomons E. Delivery of the head with the Malmström vacuum extractor during caesarean section. Obstet Gynecol 1962;19:201-203.

5. Arad I, Linder N, Bercovici B. Vacuum extraction at caesarean section - neonatal outcome. J Perinat Med 1986;14(2):137-140. https://doi.org/10.1515/jpme.1986.14.2.137

6. Pelosi MA, Apuzzio J. Use of the soft, silicone obstetric vacuum cup for delivery of the fetal head at cesarean section. J Reprod Med 1984;29(4):289-292.

7. Boehm FH. Vacuum extraction during caesarean section. South Med J 1985;78(12):1502.

8. Bofill JA, Rust OA, Schorr SJ, et al. A randomised prospective trial of the obstetric forceps versu the M-cup vacuum extractor. Am J Obstet Gynecol 1996;175(5):1325-1330. https://doi.org/10.1016 s0002-9378(96)70049-2
9. Dimitrov A, Pavlova E, Krŭsteva K, Nikolov A. Caesarean section with vacuum extraction of the head. Akusherstvo Ginekologiia 2008;47(3):3-6.

10. Sritippayawan S, Chantrapitak W. Assisted delivery of high floating fetal head: A comparison of vacuum-assisted delivery with manual extraction. Asian Biomed 2011:5(5):699-703. https://doi. org/10.5372/1905-7415.0505.092

11. National Institute for Health and Care Excellence. Caesarean section. Clinical guideline [CG132] London:NICE, 2011.

12. Spain JE, Tuuli M, Stout MJ, et al. Time from uterine incision to delivery and hypoxic neonatal outcomes. Am J Perinatol 2015;32(5):497-502. https://doi.org/10.1055/s-0034-1396696

13. Maayan-Metzger A, Schushan-Eisen I, Todris L, Etchin A, Kuint J. The effect of time intervals on neonatal outcome in elective cesarean delivery at term under regional anaesthesia. Int J Obstet Gynecol 2010;111(3):224-228. https://doi.org/10.1016/j.ijgo.2010.07.022

14. Clark SL, Vines VL, Belfort MA. Fetal injury associated with routine vacuum use during caesarean delivery. Am J Obstet Gynecol 2008;198(4):e4. https://doi.org/10.1016/j.ajog.2007.12.00

15. Bofill JA, Lencki SG, Barhan S, Ezenagu LC. Instrumental delivery of the fetal head at the time of elective repeat caesarean: A randomised pilot study. Am J Perinatol 2000;17(5):265-269. https://doi. org/10.1055/s-2000-10009

16. Wen SW, Liu S, Kramer MS, et al. Comparison of maternal and infant outcomes between vacuum extraction and forceps deliveries. Am J Epidem 2001;153(2):103-107. https://doi.org/10.1093/ aje/153.2.103

17. Johnson JH, Figueroa R, Garry D, Elimian A, Maulik D. Immediate maternal and neonatal effects of forceps and vacuum-assisted deliveries. Obstet Gynecol 2004;103(3):513-518. https://doi. org/10.1097/01.aog.0000114985.22844.6d

18. McQuivey RW, Block JE. Vacuum-assisted caesarean section. Int I Womens Health 2017;9:151-155. https://doi.org/10.2147/ijwh.s129814

19. Nakano R. Use of the vacuum extractor for delivery of the fetal head at caesarean section. Am Obstet Gynecol 1981;141(4):475-476.

Accepted 7 May 2019 